\title{
Effects of Silkworm Pupae on Bone Mineral Density in Ovariectomized Rat Model of Osteoporesis
}

\author{
Kang-Sun Ryu*, Heui-Sam Lee, Kee-Young Kim, Mi-Ja Kim, and Pil-Don Kang \\ Department of Agricultural Biology, National Academy of Agricultural Science, RDA, Suwon 441-853, Korea
}

(Received 19 April 2012; Accepted 28 June 2012)

Osteoporosis is characterized by the reduced density of mineralized bone matrix. The loss of bone has been attributed to an imbalance between bone formation and bone resorption. The silkmoth is one of the famous traditional tonic medicines. Silkworm pupa was evaluated for its antiosteoporotic activity in an ovariectomized rat model of osteoporosis. The rats were ovariectomized at 6 weeks of age and divided into 7 groups. All the groups were fed without calcium diet. The OVX rats were treated with water and silkworm powder for 6 weeks. In OVX rats, the body weight, feeding and water intake quantity did not show a significant change, but the silkworm pupa powder immediately before the eclosion of Yeonrokjam was significantly increased the bone mineral density in the femoral bone. The silkworm pupa powder increased the bone with increasing calcium in serum. These results also showed its protective action through promotion of bone formation. The silkworm pupa powder has a definite antiosteoporotic effect; similar to estrogen, it is especially effective for the prevention of bone fracture induced by estrogen deficiency. The silkworm pupa powder is a promising anti-osteoporotic agent that can prevent bone fractures in osteoporotic patients. The silkworm pupa powder does not show serious side effects because natural herbal medicine origin.

Key words: Silkworm, Silkworm pupae, Osteoporosis

\footnotetext{
*To whom the correspondence addressed

Department of Agricultural Biology, National Academy of Agricultural Science, RDA, Suwon 441-853, Republic of Korea;

Tel: +82-31-290-8518; Fax: +82-31-290-8516;

E-mail: ryuks@korea.kr
}

\section{Introduction}

Osteoporosis is a chronic, progressive disease of the skeleton characterized by bone fragility due to reduction in bone mass and possibly to alteration in bone architecture that leads to a propensity to fracture with minimum trauma (Kelly 1996). The loss of bone has been attributed to an imbalance between bone formation and bone resorption. The type of osteoporosis associated with ovarian hormone deficiency following the menopause is currently the most common cause of age associated bone loss (Arjmandi et al. 1996).

If estrogen is injected to the women in the menopause stage, it can reduce the illness caused by the unstable vasomotion and degeneration of urogenital organs and prevent osteoporosis and the symptoms related to cardiovascular activity (Maddox et al., 1998).

Osteoporosis is currently defined as "a skeletal disorder characterized by compromised bone strength, predisposing a person to an increased risk of fracture" (Wehren, 2003). Many synthetic agents, including estrogens, have been developed to treat osteoporosis, but each of them is associated with adverse events such as hypercalcemia, hypercalciurea, increased risk of endometrial and breast cancer, breast tenderness, menstruation, thromboembolic events, vaginal bleeding and hot flashes (Genant et aI. 1998; Canalis et al. 1988; Gorman and Park 2002). Hence, it would be most helpful to explore naturally occurring substances, especially of plant origin, that could prevent bone loss and are free from any adverse effects.

Epimedii Herba is an important traditional Chinese herbal medicine used widely as a tonic, aphrodisiac, and antirheumatic in China and Korea. Recently, it has been used in many Chinese antiosteoporosis formulae (Gao et al. 1999). Pharmacological studies also show that it has potential activity against osteoporosis (Li et al. 1996; Ma et al. 2002).

In Korea, several Chinese medications have tradition- 
Table 1. The various sample with silkworm grown stage, sex and varieties

\begin{tabular}{|c|c|c|c|c|}
\hline & & Growth stage and sex & Variety of silkworm & Rats \\
\hline \multicolumn{2}{|c|}{ Con } & Non-treatment & Control & \multirow{9}{*}{6} \\
\hline A & YW53F & 5 instar $3^{\text {rd }}$ days larvae $(+)$ & Yangwonjam & \\
\hline B & YW53M & 5 instar $3^{\text {rd }}$ days larvae $\left({ }^{\lambda}\right)$ & $"$ & \\
\hline $\mathrm{C}$ & YWP14F & pupae bfore the eclosion( $(q)$ & $"$ & \\
\hline $\mathrm{D}$ & YWP14M & pupae before the eclosion $\left(\delta^{\Uparrow}\right)$ & $"$ & \\
\hline $\mathrm{E}$ & YRP14F & pupae before the eclosion $(q)$ & Yeonnokjam & \\
\hline $\mathrm{F}$ & YRP14M & pupae before the eclosion $\left(\delta^{\uparrow}\right)$ & $"$ & \\
\hline $\mathrm{G}$ & AYWF & adult $\left(\begin{array}{l}(\rightarrow) \\
\end{array}\right.$ & Yangwonjam & \\
\hline $\mathrm{H}$ & AYWM & $\operatorname{adult}\left(\delta^{1}\right)$ & $"$ & \\
\hline & & Total 9 groups & & 48 \\
\hline
\end{tabular}

ally been utilized for the treatment of erection dysfunction, but their effect has never been scientifically proved until recently. It has been reported that the alcohol extract of male pupae showed that the testosterone levels in serum increased by $33 \%$, that of sperm amount increased by $41 \%$ (Ryu et al., 2002). Also this powder of silkworm pupa increased the estrogen hormone secretion(Ryu et al., 2011). The aim of this study is to evaluate the effects of silkworm pupa powder in preventing osteoporosis and ameliorating bone loss in ovariectomized rats.

\section{Materials and Methods}

\section{Animals and diets}

Six-week-old female rats of SD-strain were purchased from SAMTAKO Bio Korea and were grown. Rats were reared 6 animals to a cage and maintained at $22^{\circ} \mathrm{C}$, room temperature and $50 \%$ humidity, respectively ( $12 \mathrm{hrs}$ of light and dark cycle) with free access to rodent diet (SAMTAKO Bio Korea) and sterilized water.

When rats were grown to weight of $160 \sim 190 \mathrm{~g}$, they were divided into 9 groups ( $\mathrm{n}=6$ per group). Food and drinking water were supplied ad libitum. The blood serum was taken after fasting for $16 \mathrm{hrs}$ before the end of experiment.

\section{Preparation of silkworm according to the growth stage} The silkworms used for the experiment were Yangwonjam and Yeonnokjam that are successively preserved as the silkworm leading variety in Korea. Yangwonjam is sex-limited silkworm bred with larval marker. This variety shows body marker only on the back of female larvae, and the markers are obvious enough for the non-special- ists to separate sex casually. Yeonnokjam is green color specific variety. These silkworms were reared in the spring season of 2011 in the National Academy of Agricultural Science. The 3rd day of 5th instar, the larvae were quickly frozen with liquid nitrogen, and the pupae immediately were frozen before the eclosion, and the eclosed adults were frozen in deep freezer. These samples were subjected to fine powder prepared using a grinder mixer machine as the Table 1.

\section{Ovariectomy and administration of silkworm powder}

Ovariectomies were performed under aseptic conditions. The rats were anesthetized by intraperitonieal injection of $0.1 \mathrm{mg} / 100 \mathrm{~g}$ of mixture with ratio of 7:3 ketamine $(50 \mathrm{mg} / \mathrm{ml})$ and xylaxine $(22.4 \mathrm{mg} / \mathrm{ml})$. The ovaries were exteriorized, ligated and removed via bilateral lumbar incisions. The wound was then sutured.

After a week of acclimation, the normal group was fed the standard chow not containing the sample and water which is sterilized by ultraviolet radiation and filtered. Together with the pulverized hard ford purchased from Samtaco. Co (Osan, Korea) in Wonwang Mill (Jeonju, Korea), $250 \mathrm{mg} / \mathrm{kg}$ of frozen silkworm powder was administered to forty eight rats for 6 weeks except for control group. We measured the average eating rate during the entire week since the administration of freezing driedsilkworm powder.

\section{Serum and bone parameters}

After the last administartion, whole blood was collected from the rat's abdominal vein by surgery with ether anesthesia. The collected bones were treated with powder at $600^{\circ} \mathrm{C}$ in the Maffle's furnace. Blood samples for serum by centrifugation at $3000 \mathrm{rpm} 15 \mathrm{~min}$ were analyzed at UB Medical Institute. 


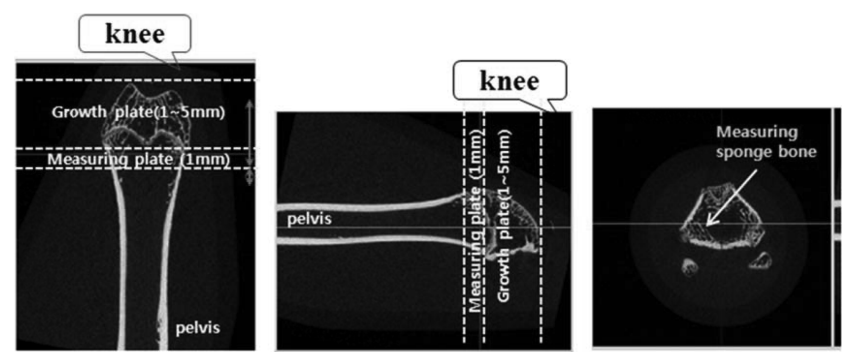

Fig. 1. The measuring plate is located $1 \mathrm{~mm}$ volume below $5 \mathrm{~mm}$ from knee.

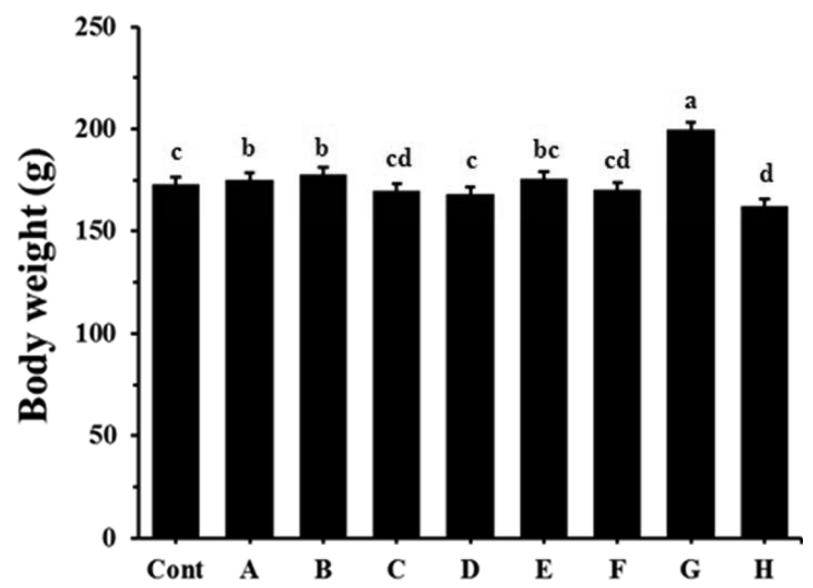

Fig. 2. Effects of Silkworm on changes of body weight in the ovariectomy-induced osteoporosis animal model. Data are means \pm SE $(n=5)$. Bars with different letters from the control are significantly different $(\mathrm{p}<0.05)$.

\section{Measurement of bone mineral density (BMD)}

Bone specimens were obtained from the right and left femur of sacrificed rats. The measuring plate was $1 \mathrm{~mm}$ volume below $5 \mathrm{~mm}$ from the knee(Fig. 1). The bone mineral density(BMD) tests were performed on the proximal femur by using NFR polaris-G90(Nanofocusray Co.), 2dimensional bone morphometric analysis by custom computer program, and 2D bone structure anlaysis by using micro-CT. Statistical analysis was carried out for the correlation between bone morphometry, micro-CT and BMD.

\section{Statistical analysis}

Statistical analysis of the data from the animal experiments in this report was performed by the one-way analysis of variance (ANOVA) followed by the Duncan test for multiple comparisons. A $p$ value less than or equal to 0.05 was considered a statistically significant difference.

\section{Results}

\section{Body weight}

Our analysis focused on the comparison of changes in body weights between the treatment group and the control group after 6weeks of feeding silkworm powder. The YWAF group showed an increased body weight greater than the control group. But these results did not show much difference between the two groups despite the time changes (Fig. 2). No apparent weight loss was observed in the control group which proves that the nutrition was not a relative matter.

Feeding the silkworm sample from various growth stages is very safe in animal tests since it had no influence on the weight.

\section{Calcium and phosphorus in femoral and in serum}

The contents of calcium and phosphorus in the femur of
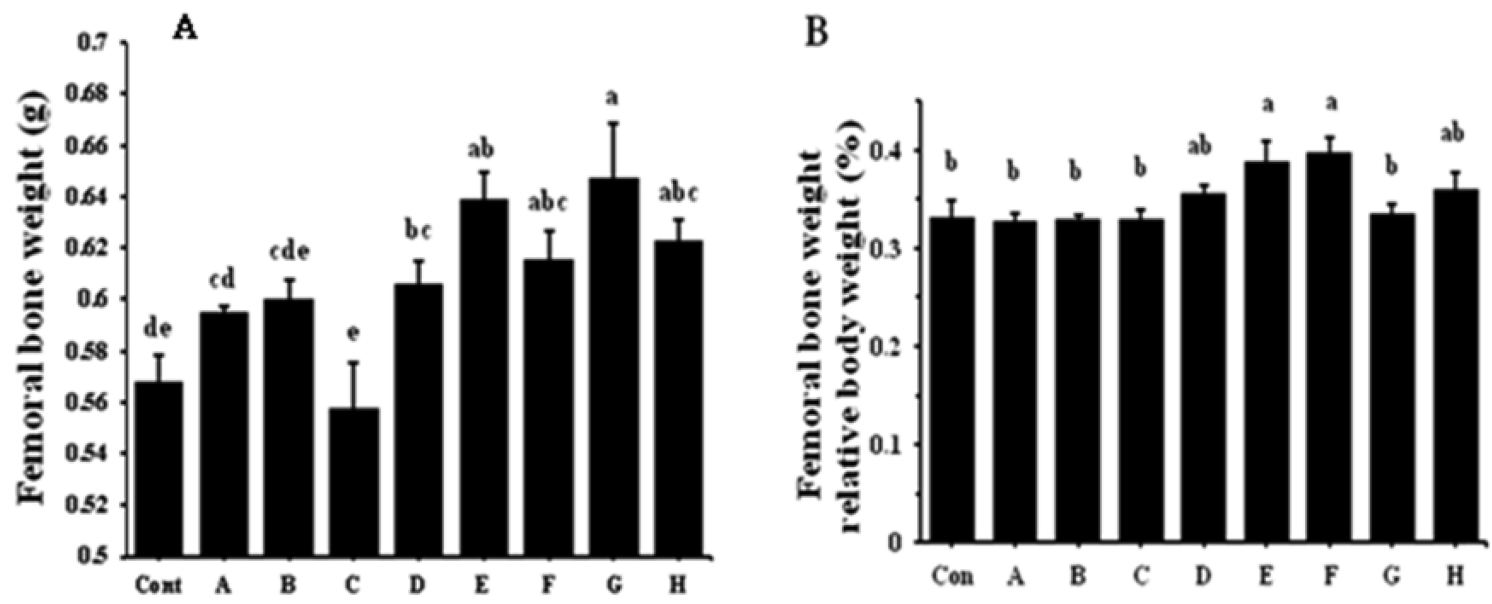

Fig. 3. Effects of silkworm on absolute(A) and relative body weight (B) of femoral bone weight in ovariectomy-induced osteoporosis animal model. Data are means $\pm \operatorname{SE}(n=5)$. Bars with different letters from the control are significantly different $(p<0.05)$. 
A

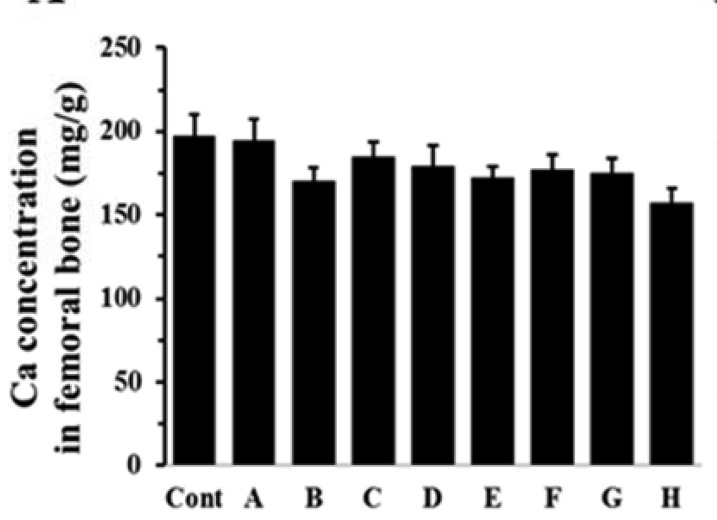

B

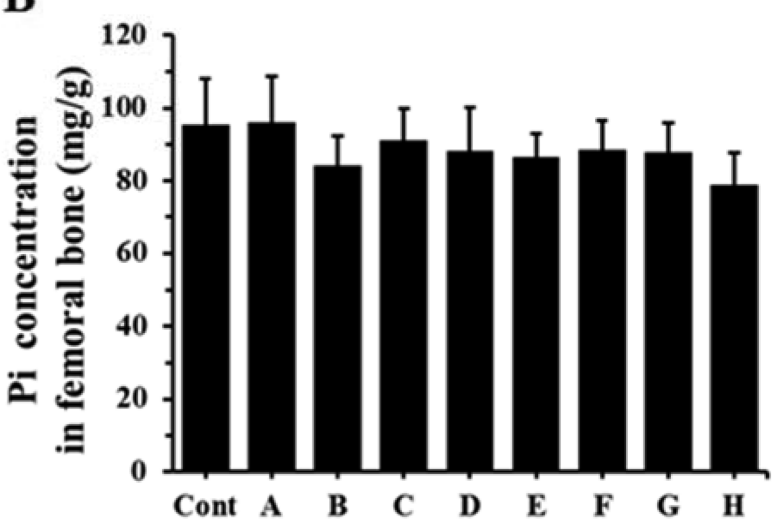

Fig. 4. Effects of silkworm on changes in calcium (A) and phosphorus (B) concentration in femoral bone of ovariectomy-induced osteoporosis animal model. Data are means $\pm \operatorname{SE}(n=5)$.
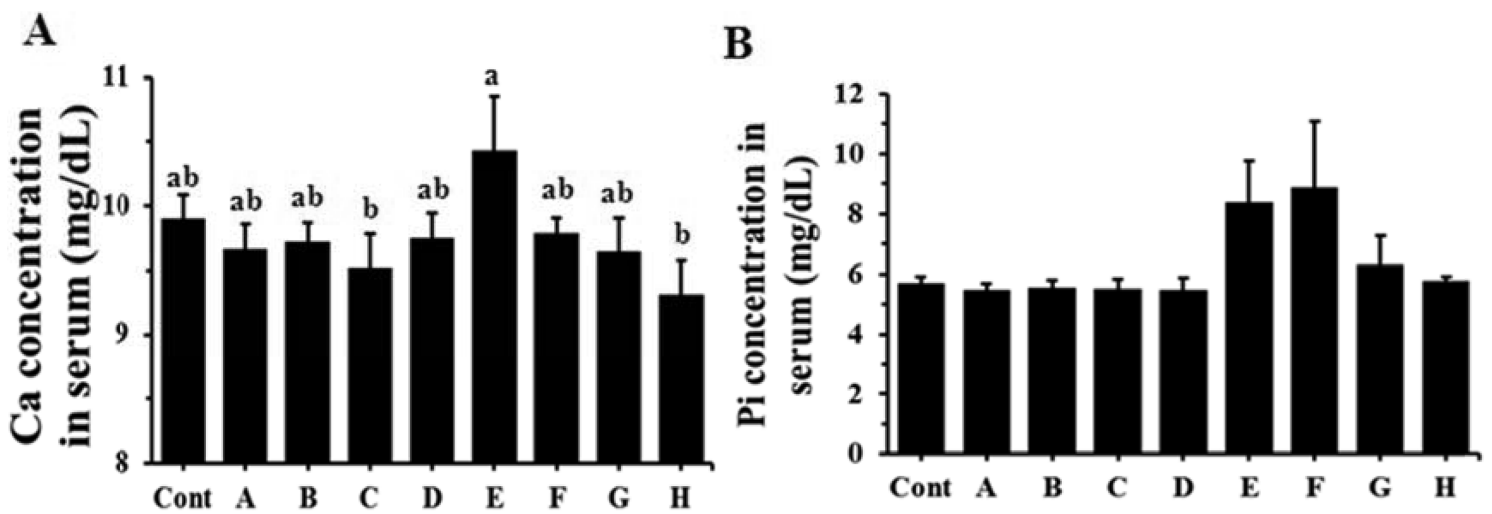

Fig. 5. Effects of silkworm on changes in calcium (A) and phosphorus (B) concentration in serum of ovariectomy-induced osteoporosis animal model. Data are means $\pm \mathrm{SE}(\mathrm{n}=5)$. Bars with different letters from the control are significantly different $(\mathrm{p}<0.05)$.

the experimental group did not differ much from the control groups(Fig. 4). However, the amount of calcium in the blood increased only in group E, whereas the level of phosphorus in the blood of groups $\mathrm{E}$ and $\mathrm{F}$ increased more than the control group(Fig. 5).

\section{Bone mineral density}

The calcium free diet was fed for six weeks after ovariectomized. At the same time, the rats for osteoporosis model was fed with silkworm-extract ingredients. We measured BMD in femur. BMD increased in the experimental groups E, B, G, and H. Particularly, it increased the most in group E(Fig. 6). Fig. 7 shows the picture of $1 \mathrm{~mm}$ volume below $5 \mathrm{~mm}$ from the knee. We excluded the growth plate located at the bottom of the knee and the compact bone. We instead measured the BMD of the sponge bone in the compact bone. Sponge bone has disappeared as the osteoporosis progressed. Thus, comparison of the bone density in sponge bone becomes a

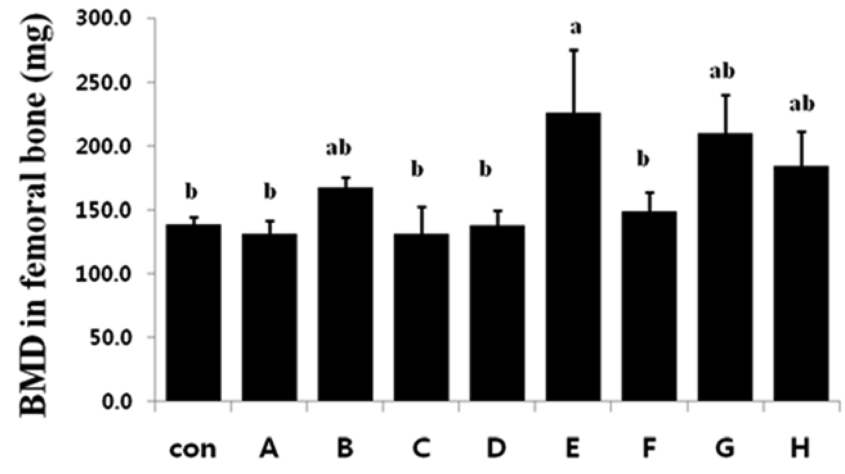

Fig. 6. Effects of silkworm on changes in bone mineral density in femoral bone of ovariectomy-induced osteoporosis animal model. Data are means \pm SE $(n=3)$. Bars with different letters from the control are significantly different $(\mathrm{p}<0.05)$.

measure of BMD increase. BMD was far more increased in group $\mathrm{E}$ than the control group. 

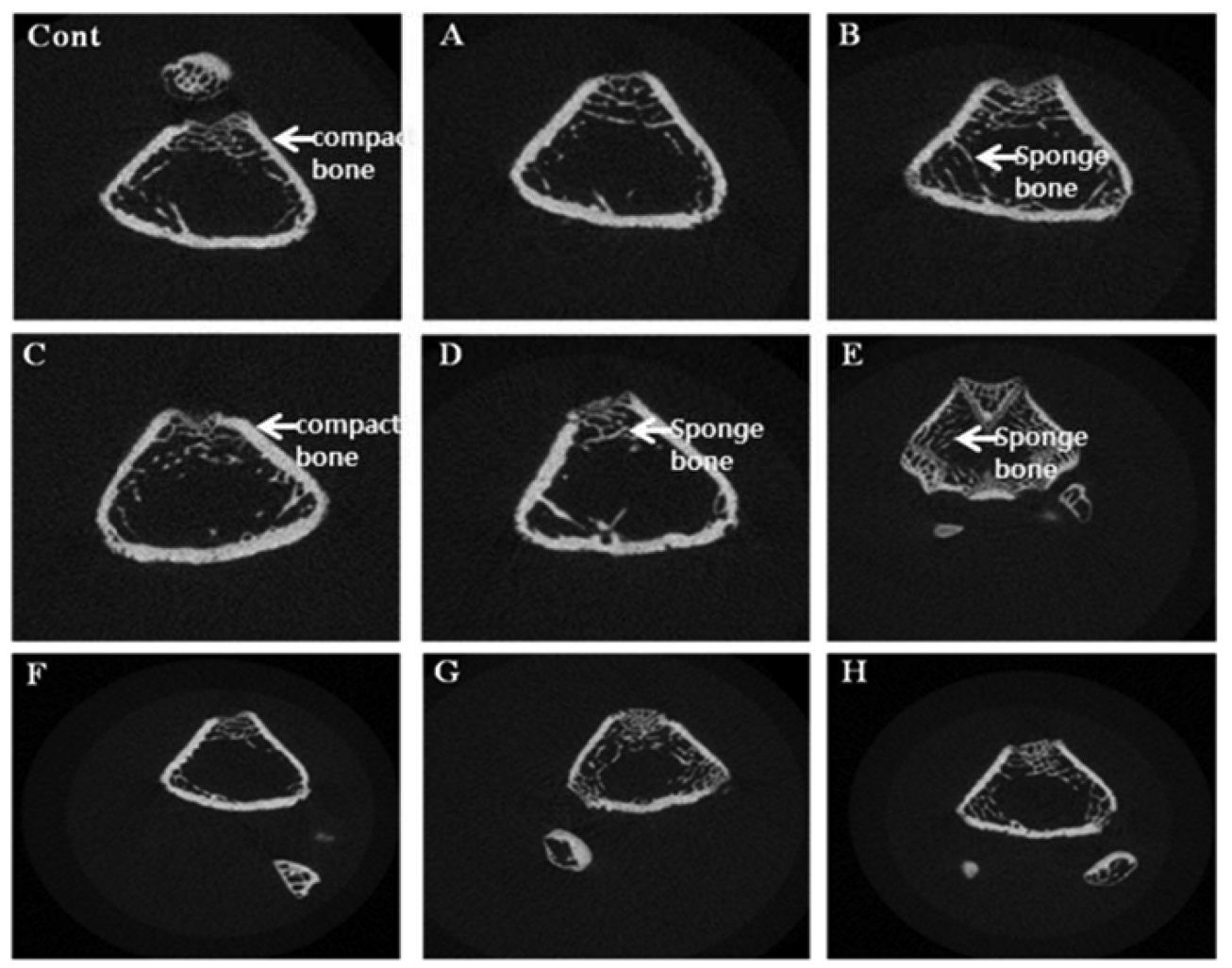

Fig. 7. Effects of silkworm on changes in bone mineral density image in femoral bone of ovariectomy-induced osteoporosis animal model.

\section{Discussion}

Our study clearly demonstrates the usefulness and beneficial effects of silkworm pupae in the prevention of bone loss induced by ovariectomy. Weight was the highest in group $\mathrm{G}$ and the lowest in group $\mathrm{H}$. However, it did not show a significant change. The weight of the femur relative to the weight similarly increased in groups of D, E, F, G, and H compared to the control groups. The weight of calcium and phosphorus in the femur in the control group did not differ from that of experimental groups. The weight of the bone's calcium and phosphorus per weight of the femur was also not different between the two groups. The content of calcium in the blood tended to increase in group E compared to control group while that of phosphorous did not show any statistically significant changes. In this study, BMD was high in groups E, G, H, $\mathrm{B}$, and $\mathrm{F}$ compared to that of the control groups. Particularly, the BMD increase in group E was statistically significant. increase of BMD in the comparison among groups was also statistically significant. This indicates that feeding of group $\mathrm{E}$ among silkworm-extracted ingredients maintains the content of calcium in the blood while bone absorption in the femur is in progress. It contributes to the bone formation by increasing the activity of osteoblasts and by preventing the reduction of BMD and weight of the femur. Therefore, the process of how group E contributes to the prevention of osteoporosis will be a valuable resource to study further.

Taken together, our study has shown that silkworm pupa significantly contributes to the prevention or treatment of bone loss induced by ovariectomization in rats. Furthermore, the results clearly indicate that silkworm pupa is a potential alternative therapeutic agent and may be tested in humans as an antiosteoporosis therapy.

\section{Acknowledgement}

This work was supported by a grant from the next generation BioGreen 21 Program (Project No. PJ008081012012), Rural Development Administration, Republic of Korea.

\section{References}

Arjmandi BH, Alekel L, Hollis BW, Amin D, Stacewicz Sapuntzakis $\mathrm{j}$, Guo P (1996) Dietary soybean protein pre- 
vents bone loss in an ovariectomized rat model of osteoporosis. J. Nutr 126, 161-167.

Canalis E, McCarthy T, Centrella M (1988) Growth factors and the regulation on bone remodeling. J. Clin. Invest. 81, 277-281.

Einhorn T(1992) Bone strength: the bottom line. Calcif. Tissue Int. 51, 333-339.

Gao SQ, Fu DX, Zhang HM (1999) Advances in the study on the treatment of osteoporosis with Herba Epimedii and its compound prescriptions. China J. Chin. Mater. Med. 24, 249-251.

Genant HK, Baylink DJ, Gallagher JC (1998) Estrogens in the prevention of osteoporosis in postmenopausal women. Am. J. Obstet. Gynaecol. 161, 1842-1846.

Gorman C, Park A (2002) About hormones. Time July 22, 3637.

Ke HZ, Chen HK, Simmons HA, Qi H, Crawford DT, Pirie CM, et al (1997) Comparative effects of droloxifene, tamoxifen, and estrogenon bone, serum cholesterol, and uterine histology in the ovariectomized rat model. Bone 20,31-39.

Kelly PJ (1996) Is osteoporosis a genetically determined dis- ease? Br. J. Obstet. Gynaecol. 103, 20-27.

Li QN, Liao JM, Wu T, Huang LF, Liang NC (1996) Epimedium sagittatum Maxim, preventing hormone- induced osteoporosis in rats. Chin. Pharm. J. 31, 467-470.

Ma HP, Jia ZP, Ge X (2002) Studies on the theraputic effect of total flavonoids of Herba Epimedii on experimental osteoporosis in rats. Huaxi J. Pharm. 17, 163-167.

Maddox RW, Carson DS. Barnes CI (1998) Estrogen and postmenopausal women, US Pharmacist 23, 141-150.

Ryu KS, Ahn MY, Lee HS, Kim IK, Kim JW. Kim SH, Choi JH (2002) The tonic effect of the extract from male silkworm(Bombyx mori L.) pupae on rats. Int.J.Indust. Entomol. 5(1), 123-126. 2002.

Ryu KS, Lee HS, Kim KY, Kim MJ. Oh HG, Kang PD (2011) Secretion Effect of Estrogen with Sex and Growth Stage of Silkworm (Bombyx mori L.). Int.J.Indust. Entomol. 22(1), 17-20. 2011.

Wehren LE (2003) The epidemiology of osteoporosis and fractures in geriatric medicine. Clin. Geriatr. Med. 19, 245-258, 2003. 